\title{
Analysis on Evolution Characteristics of Chinese Characters*
}

\author{
Linying $\mathrm{He}$ \\ Xingtai University \\ Xingtai, China 054001
}

\author{
Zhanping Zhang \\ Xingtai University \\ Xingtai, China 054001
}

\author{
Linna $\mathrm{He}$ \\ Xingtai Siyuan Experimental School \\ Xingtai, China 054001
}

\begin{abstract}
The glyph structure of Chinese characters are developed towards simplification, semiotics, standardization, and beautification. Simplification is the general development direction of Chinese character structure; symbolization is a result of simplification, which in turn facilitates simplified processes; Standardization is the purpose of the development of Chinese character structure; Beautification is the realistic goal pursued by Chinese character structure; Standardization also monitors the beautification, so that it must not wander too far.
\end{abstract}

Keywords-Chinese character structure; simplification; beautification; standardization; symbolization

\section{INTRODUCTION}

The evolution of Chinese character structure is not the change of aimlessness and direction, it follows the general rule of the change of Chinese characters, but also shows its own personality characteristics. In this paper, the characteristics of the evolution of Chinese character structure are summed up and analyzed on the basis of the research of the former.

\section{SIMPLIFICATION}

The simplification of the trend, the whole process of the Chinese character, has been in existence since the Chinese character. The simplification of the structure and the evolution of the font are simultaneous. That means, one type of font replaces another type of typeface, which is easy to write instead of difficult to write, and simple structure instead of complex structure. Specific implementation ways are as follows:

\section{A. It Achieves by Compliance with "Writing Physiology"}

As a tool for language communication, Chinese characters are mainly used for the purpose of application. So the first consideration should be how to make the writing more convenient, and we have the principle that convenience for

* This article for the ministry of education humanities and social science research youth fund projects Dynamic Inspection of Regular Script Font Changes of Inscriptions from the Angle of Writing (Project Number: 16 YJC740025) phased research results. hand will survive and the others will die, the principle of choosing the direction of the line with the physiological habit, and thus derive the five basic strokes of Chinese characters. This evolution process is also a simplified process. The specific performance is that the writing sequence of words is more explicit and the internal structure is more concise. Compared with ancient Chinese characters, the transformation from "painting" to "writing" is realized.

Such as “術”: Xiao Zhuan 徒, after the change of the official script Li as 政 (JingJun Tablet), “很”, the word made of 1 , is complicated and not easy to write. The official script Li turns them into “术” and “宁”, simple and easy to write. Such as “禾”, Xiao Zhuan $\Psi$, after the change of

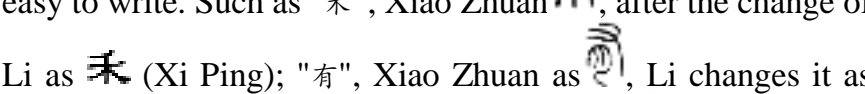
(Yin Zhou Tablet); “昔”, Xiao Zhuan as 菖, Li changes it as (Xi Ping Tablet); “毛”, Xiao Zhuan as †, Li changes it as (Kong Biao Tablet); All this is due to the simplification of glyphs that conform to the physical habit of writing.

\section{B. It Is Realized by Saving and Reducing}

Such as “夙”, Xiao Zhuan as 19 , 夕 means the night, 车( jí) means holding things by hands. Do things up just before dawn, showing earlier, official script $\mathrm{Li}$ is simplified to $\mathbf{7}$. (North Sea Jing Jun Tablet). It makes Xiao Zhuan “肃” produce into “凡”, resulting in simplified glyph. The same examples: “恐”,

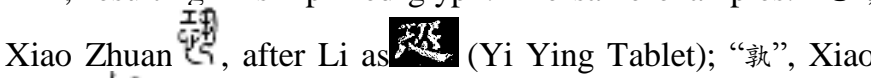

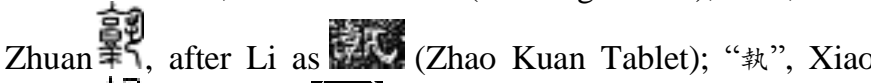
Zhuan ${ }^{4}$, after Li as (Shi Men Tablet). 
There is also the simplification of a batch of Chinese characters through the reconstruction. Such as metal “定” simplified as “远”; metal “水” simplified as “;”; metal “心” simplified as “小”; metal “邑” and “卓” simplified as “””.

\section{It Is Achieved Through A Fraud}

For example, the characters from the mental 月, 肉, 丹, 舟 are fraud after Li as “月”, such as $\overbrace{(\text { mental } 月) ~} \mathbf{F}$ (Xi Ping Tablet) ;

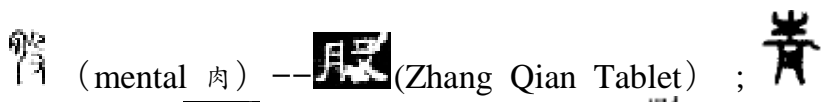
(mental 丹) (Yang Zheng Tablet); 影 (mental 舟) (Xi Ping Broken Stone) . It is a simplified form to reduce the type of Chinese characters by making it easy to confuse the components.

\section{It is Achieved Through Adhesion}

For example, “史” Xiao Zhuan as 毒, Shui Script as $(24 \cdot 19)$, the upright and the curving curve of the form is attached to a stick, and then a series of steps as 岁 (Crossbar Book 175), (Xi Chui Script53. 3) ; the inscriptions on the tablets were further improved by the straight and square strokes as (Yin Zhou Tablet) with a more simplified structure.

\section{STANDARDIZATION}

The Standardization is to "eliminate the foreign bodies and each word tends to be written in a certain way," [2], the final target of Chinese character development. Any type of font has to go through the process from occurrence, development, to maturity, final specification, and official script $\mathrm{Li}$ is no exception. According to the script of script in Han dynasty, there are several different ways of writing a word, which means that there are many different words. It is not hard to understand the reasons:

\section{A. It's a "Spontaneous Movement" Created by the People, and Not Just an Official Plan, and then to Reinforce it}

In this way, the evolution of the zigzag will not be able to behave in a formal manner, and naturally there will be different variants.

A foreign body caused by the difference of the character style is converted. For example, “昔”, oracle as ( 1968) , "From the mental 日 and 䄈, Ye Yuensen regards 被 as flood, that is, the ancient word 4 ft. The ancient people almost never forget the flood disaster, so the formation of the word. Let's take the day of the flood." Xiao Zhuan as Zhou Wen as 䇫. Because of the different character style, there are two ways of writing during the Li changes, such as

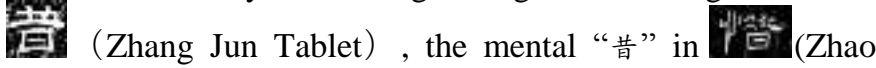
Hou boy Tablet). Another example “明”, Xiao Zhuan as “이, , according to this changed as (Yi Ying Tablet) off (West Yue Mountain Temple Tablet); in ancient article as “ㅇ)), , and decided as Another example “风”, it can be decided as 虍 (Cao Quan Tablet) according to Qin Zhuan “" $\sqrt{2}$ (Liu Xiong Tablet) according to six countries ancient articles.

Transferred to change the style of the same loyal degree caused by different variants. For example, “喪”, Xiao Zhuan 整

as “四, the mental “哭” and “亡”, is just like the meaning: death, and like the sound "Wang". When the Li changes, according to different understanding and custom, produce different writing, such as basically same with the Xiao Zhuan (Chao Hou Guy Tablet), 䢦 straight in the middle (The Third Year in Jian Ning Tablet), Jian Ning Tablet), in an omitted way (Cao Quan Tablet). Another example, “散”, Xiao Zhuan as , meaning the mixed meat with the mental 肉 and the sound 枝. After the Li change, components “文”, “没” mixed together. Therefore, there are some parts that retain the small seal shape (Zhao Kuan Tablet), and also the adhesion of the (Stone Door Tablet). Like “禽”, Xiao Zhuan as 饧, after the change of the $\mathrm{Li}$ is loyal to the small seal (Tong Bai Huai Yuan Temple), also has the simplified glyph (Zhang Qian Tablet).

\section{B. Since Most of the Inscriptions Are Displayed in the Public and Are Viewed by Others, the Composition of Calligraphy Is Naturally Incorporated into the Creation.}

In this way, it is possible to create a large number of tablets by consciously adding and subtracting strokes, changing components and reorganizing structures in order to express individuality and to seek different ideas.

Add and subtract strokes. Stroke reduction, such as: 属: 굴

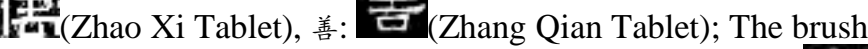

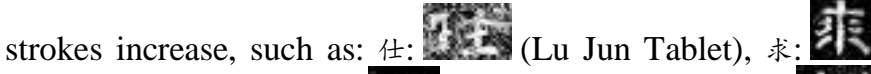
(Yang Tong Tablet), 名: 名 (Cao Quan Tablet), 副: 舅] (Cao Quan Tablet).

Mixing of components. Such as the components, 口 and ム, 刀 and 力, the mental of 草 and 竹, 没 and 父, 穴 and ${ }^{\circ}$, 
又 and 寸, 前 and 羊, 凡 and 丸, 山 and 止, 氵 and ; , these are commonly used components of a very strong construct. There are many characters involved, and their mixing is bound to cause a large number of foreign bodies. Such as: 留: Jun Tablet), 稙 (Zhang Qian Tablet); 松: (Li Qi Tablet), 桘 (Fei Zhi Tablet).

Through the above analysis, it can be seen that the emergence of the tablet is a manifestation of the conscious or involuntary behavior of people using Chinese characters. The simplification trend is important, but when it does affect people's normal reading, it is bound to be regulated. According to the rule of simplification and optimization of Chinese characters, the "positive body" is determined to ensure normal social communication, while others are preserved as foreign characters. "Each" official body "is stable for a long period of time, guaranteeing the circulation of the word in the whole society. But it does not prevent the creation of new 'another body', and when a new ' another body' gains an advantage over the old 'body', it may be replaced by a new 'body'. [2] In a word, the evolution of the Chinese character form and the change of the internal structure are developed and shaped in the standard of foreign characters.

\section{SYMBOLIZATION}

In discussing the character of Chinese characters, Mr. Qiu Xigui mentioned the "character", which means "the symbol used in the text is called 'character'." The "characters" can be divided into three categories: the signifier, the note and the mark. (The character that is associated with the word in meaning is the signifier, the phonetic connection is the note, and there is no contact between the voice and the meaning.) There are different characters used at different stages. Specifically, "Chinese characters are in the early stage of higher hieroglyphics (generally speaking before the Western Zhou Dynasty), which is basically a writing system using the signifiers and notes. Later, along with the changes in glyphs and phonetics and meanings, it gradually evolved into a kind of writing system (the formation of official script can be regarded as the symbol of this evolution) using the signifiers (mainly the meanings), notes and symbols. "Symbolization", as we call it, is the general term for Chinese characters, such as abstract, notes and marks. When people writing, no longer consider what text symbols represented as "like", but write it as a symbol of difference significance, thus prompting the birth of purely abstract symbolic words.

For example, “前”, there are two characters in Spoken Book, one is “歨” with the mental “止”, “芦 means progressing without walking. Keep static on the boat." The others is “剪” with the mental “刀”. “族 means cutting in a neat way, with the knife, sounding like 歨”. From the character meaning, “前” is the same as from with the $\frac{A^{\prime}}{5}$. That is because which means

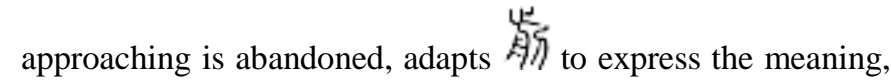
then create another character “剪” to show cutting neatly. When the transformer is changed, the “前” component “舟” and “止” are gradually written as “月” and “项” under the action of the direct and reverse nature of the writing physiology. Examples are as follows:

$$
\begin{aligned}
& \text { (Xiao Zhuan) } \rightarrow \text { 前(Sleeping Script 54.43) } \rightarrow \text { 前 }(\text { Lao Zi } \\
& \text { after Jia208) } \rightarrow \text { (Cao Quan Tablet) }
\end{aligned}
$$

From the structure of the change of the $\mathrm{Li}$, the structure of "月" and " $"$ have lost the structure and become meaningless symbols, so the "pictographic" of Chinese characters is no longer so important. As long as it conforms to the writing habit and can be recognized by people, it can obtain long-term vitality and finally obtain legal status.

Another example, “申”, Jin Script as $\overline{\boldsymbol{\varepsilon}}$, in the shape of 细

lightning; Xiao Zhuan as 1 , losing the original intention, but there are clues can be found; transferred to change according to specification will be flat founder's words when writing "申", the shadow of the lightning was gone, completely into a symbol.

In conclusion, under the constraints of this principle, and the power of writing and writing physiology, the structure of the script is in the process of the natural, undetectable, symbolic process.

\section{BEAUTIFICATION}

As a communicative tool, Chinese characters are easier to write and standardize in order to identify them. On top of that, people will look for beauty in the shape to satisfy the aesthetic psychological needs. The image of the characters reflected by the character of the Chinese character, which is changed by the $\mathrm{Li}$, is gradually separated from the image. This means that the pictographic characters will be removed from the historical stage and will be replaced by highly abstracted symbolic characters. In this way, the pursuit of Chinese characters is more aroused. If writing convenience is the original purpose of the transformer, then the pursuit of glyphs is the ultimate goal. However, the Han dynasty inscriptions are the main achievement of this ultimate goal.

The inscriptions in the Han dynasty, such as the stone classics, the achievements, the monuments, the ancestral tombs, and so on, most of them is in the public, or for people to imitate, watch, with a view to the spread of the ancient. Since these official script works with "social openness", then the calligrapher must comply with the social and cultural background in the required specifications, with a rigorous, dignified and beautiful writing tablet as soon as possible. It should be done so as to be serious, formal and dignified. Of course, this kind of conscious beautification is gradually highlighted and strengthened with the development of official script.

In the early days of the script, the script in the western Han dynasty was in the early stage of the transformation. The 
official script of this period focused on the transformation and the structure of seal script, such as The Inscription on the Tombstone of the Huo Qubing, The Carved Stone of the Stone, The Stone of Luo Xiaoyu, and The Stone of the La Zihou. In the early period of the eastern Han dynasty, the characters of official script were marked by the characteristics of wave selection and division. For example, The Article about Zhang Wensi Build Stone Palace for His Father, and almost every word is used to outline the wave tail with a single line, this kind of deliberate emphasis showing the people's aesthetic pursuit. In the middle and late eastern Han dynasty, the symbol of "the tail of the silkworm" and "one wave and three fold" as the symbol of balance and symmetry as the typical characteristics of the mature official script, such as Cao Quan Tablet, Sacrificial Vessel Tablet.

There are also examples of the change of organizational structure in calligraphy. (This is one of the reasons for the inscription, which has been discussed in the previous section and is illustrated in a few more cases.) It's like “氏”, in Feng Jun In Ji Zhou Tablet, Ein Zhou Tablet, and the “氏”, the right shoulder is a little bit more, a decorative thing; “土”, 斯 in Feng Jun In Ji Zhou Tablet, in The White Stone Tablet, 城 with the mental “土” in Cao Quan Tablet, all have a little point. “在”, in the presence of a monument, to the beauty of symmetry, adds a little bit more point. It's like “材”, Fist in Shi Chen Tablet, “财”, Huai Yang, and the “才”, adds a little point, and which's impossible to find the genetic clues from the seal and the text, There seems to be no more reasonable explanation than to beautify (to break the "horizontal", or to the left and right structure). Again, such as “在”, in Zhang Qian Tablet, left a dash or short shaft, have the effect of harmonic, balance the font, this structure is accepted by the later regular script, and in use today. “美”, 差 in Cao Quan Tablet, in The West Narrow Ode, has the mental “大” department of the long horizontal writing two points; “麦”, ह

as in The West Narrow Song, adds a bit more at the end pen; “亞”, adds a line to fill in the shape of the glyph. There are many more examples. In the written and early engraving of the stone, you can hardly see the structure, which tells you that when the pursuit of convenience is no longer the first purpose of writing, the pursuit of beauty will be revealed.

Of course, beautification is not only a complex structure, but sometimes it also saves strokes. For example, for “喜”, 놀류. in the Xi Ping Tablet, the two points on the long horizontal line are a little bit, which is consistent with the superstructure, the simple structure and the beautiful shape. “盈”, as in Zhang Jun Tablet; “久” in the character “乃” is omitted and symmetry is not crowded. In a word, the beautification principle is applied to the later writing of official script, which can be seen everywhere in the inscriptions of the eastern Han dynasty. But when the form of beauty is emphasized to a certain extent, it falls into the stylized fence; instead, a simpler and more elegant font is needed to explain the fact that regular script replaces official script.

\section{CONCLUSION}

The glyphs of Chinese characters are developed towards simplification, semiotics, standardization, and beautification. Of the total development direction of simplified Chinese characters structure, is written by almost all the common consciously and unconsciously done; Symbolization is a result of simplification, which in turn facilitates simplified processes. Normalization is the purpose of the development of Chinese character structure, which is sure to simplify the results while stopping the confusion caused by simplification. Beautification is the realistic goal pursued by the Chinese character structure. Sometimes it is the opposite of simplification. Standardization also monitors the beautification, so that it must not wander too far. In short, these aspects interact with each other, restrict each other, and combine together to form the main keynote of the change of Chinese character structure.

\section{REFERENCES}

[1] Liang Donghan, 1959 The Structure and Rheology of Chinese Characters, Shanghai Education Publishing Press.

[2] Lin Yun,1986 A Brief Introduction To The Study of Ancient Texts, Ji Lin University Press.

[3] Xu Zhongshu,1989 Oracle Dictionary, Si Chuan Dictionary Press.

[4] Qiu Xiyao,2004 A Brief Introduction To Literature, Commercial Press.

[5] Bao Xianlun,1985 The Influence of Writing on the Evolution of Chinese Characters, Journal of Shao Xing University Of Arts And Sciences (Social Science Edition) no. 4. (Mailing Address: 054001, Xing Tai College, He Bei Province) 